\section{Graduação em saúde: oferta e estratégias para o fortalecimento da regionalização do Sistema Único de Saúde}

\author{
Undergraduate studies in health: supply and \\ institutional strategies in the context of the \\ Brazilian Unified National Health System \\ regionalization
}

\author{
Grado en salud: oferta y estrategias \\ institucionales contextualizadas respecto \\ a la regionalización del Sistema Único de \\ Salud brasileño
}

\section{Resumo}

Este artigo objetiva descrever o panorama de distribuição territorial da oferta de formação em saúde, bem como identificar as estratégias para o fortalecimento da regionalização adotadas pelas instituições de ensino que ofertam cursos de saúde e as mudanças verificadas no entorno regional em função dessas instituições. Trata-se de estudo descritivo-exploratório, do tipo estudo de casos múltiplos, desenvolvido em âmbito nacional no período de dezembro de 2015 a setembro de 2016. Foram analisados dados secundários de oferta de formação em saúde e foram entrevistados 68 gestores de ensino de cursos de graduação em saúde, cujos depoimentos foram submetidos a análise de conteúdo. Percebe-se um aumento de equipamentos para formação em saúde em regiões e municípios de menor porte, não obstante a concentração em locais com maior desenvolvimento socioeconômico. Politicas de acesso ao ensino superior de estudantes vêm sendo empreendidas, na perspectiva de promover a provisão e fixação profissional do entorno onde se localizam os cursos da saúde. Constata-se que a presença da universidade promove desenvolvimento regional e tem potencial para o fortalecimento da regionalização da saúde.

Regionalização; Recursos Humanos em Saúde; Educação Superior;

Universidades
Celia Regina Pierantoni 1

Carinne Magnago 1,2

Swheelen de Paula Vieira 1

Márcia Silveira Ney 1

Rômulo Gonçalves Miranda 1

Sabado Nicolau Girardi 3

doi: 10.1590/0102-311X00066018

\author{
Correspondência \\ C. Magnago \\ Instituto de Medicina Social, Universidade do Estado do \\ Rio de Janeiro. \\ Rua São Francisco Xavier 524, Rio de Janeiro, RJ \\ 20550-900, Brasil. \\ carinne.mag@gmail.com \\ 1 Instituto de Medicina Social, Universidade do Estado do \\ Rio de Janeiro, Rio de Janeiro, Brasil. \\ 2 Faculdade Meridional, Passo Fundo, Brasil. \\ 3 Núcleo de Educação em Saúde Coletiva, Universidade Federal \\ de Minas Gerais, Belo Horizonte, Brasil.
}




\section{Introdução}

O acesso aos serviços de saúde é prerrogativa indispensável para o êxito dos sistemas de saúde, os quais devem ser organizados de modo a responder às necessidades da população usuária, em coesão, portanto, com os aspectos sociodemográficos e epidemiológicos do território 1,2 .

Partindo dessa premissa e considerando a dimensão territorial do Brasil, a estruturação regionalizada do Sistema Único de Saúde (SUS) é assumida como estratégia capaz de assegurar uma ação mais eficaz do Estado, em prol da garantia do direito à saúde previsto constitucionalmente há 30 anos 3 .

A organização de uma rede regional ainda se constitui como um desafio persistente, especialmente caracterizado pela desigualdade de acesso e pela fragmentação da atenção à saúde 1,2. Esse cenário se deve, muito em parte, às iniquidades, quantitativas e qualitativas, de oferta de recursos humanos em saúde que não foram superadas com a expansão do ensino superior, nem com o direcionamento do processo de formação em saúde pelas Diretrizes Curriculares Nacionais 4.

Ao longo dos últimos anos, iniciativas foram aplicadas a fim de não só superar os déficits existentes quanto à oferta de profissionais para uma adequada distribuição no país, mas também de induzir mudanças curriculares com vistas à reorientação da formação, a exemplo do Programa Nacional de Reorientação da Formação Profissional em Saúde (Pró-Saúde), o Programa de Valorização do Profissional da Atenção Básica (Provab) e, mais recentemente, o Programa Mais Médicos (PMM).

Tais iniciativas assumem como desafio a criação de dispositivos político-pedagógicos que articulem trabalho e formação em saúde, na perspectiva do desenvolvimento de habilidades e competências multiprofissionais adequadas às realidades locorregionais e ao fortalecimento dos princípios do Sistema Único de Saúde (SUS) 4 .

Esse movimento de construção de uma política de formação assentada nas demandas da população apresenta conquistas, descontinuidades e desafios não superados que exigem o fortalecimento e/ou a criação de novas condutas de reorientação da oferta e do processo de ensino em saúde 5 , o que requer informação atualizada, adequada e oportuna sobre as mudanças operadas nos contextos regionais.

Pelo exposto, este artigo objetiva descrever o panorama de distribuição territorial da oferta de formação em saúde, identificar as estratégias para o fortalecimento da regionalização adotadas pelas instituições de ensino que ofertam cursos de saúde e apresentar as mudanças verificadas no entorno regional em função dessas instituições.

Pretende-se, com este artigo, contribuir com a discussão da oferta de formação em saúde contextualizada com as necessidades regionais do país, como estratégia de fomento ao desenvolvimento do SUS regional. Pretende-se também que este estudo seja um fator contributivo para o desenvolvimento regional, o qual sustenta a ideia de processo de mudança social da região, na garantia de universalização e de exercício efetivo dos direitos humanos individuais e coletivos, incluindo a saúde e a educação 6 .

\section{Método}

Este estudo se configura como pesquisa qualiquantitativa, descritiva, exploratória, do tipo estudo de casos múltiplos, desenvolvido em âmbito nacional, no período de dezembro de 2015 a setembro de 2016.

A abordagem quantitativa foi empregada para a descrição do panorama de oferta de formação de recursos humanos em saúde no Brasil, por meio de dados secundários disponibilizados pelo Instituto Nacional de Estudos e Pesquisas Educacionais Anísio Teixeira (INEP) do Ministério da Educação (MEC) 7 e processados pelo Sistema de Indicadores das Graduações em Saúde (SIGRAS), ferramenta on-line que gerencia dados e informações sobre as instituições de ensino superior que ofertam cursos em saúde (Observatório de Recursos Humanos em Saúde, Estação de Trabalho, Instituto de Medicina Social, Universidade do Estado do Rio de Janeiro. Sistema de Indicadores das Graduações em Saúde. http://www.obsnetims.org.br/sigras/, acessado em 30/Set/2017).

Neste estudo, os dados capturados para a análise do panorama foram os relacionados aos cursos de Biomedicina, Educação Física, Enfermagem, Farmácia, Fisioterapia, Fonoaudiologia, Medicina, 
Nutrição, Odontologia, Psicologia, Saúde Coletiva e Terapia Ocupacional. Para uma análise por regiões de saúde, foram consideradas as informações das graduações em Enfermagem e Medicina, do período de 2000 a 2014. Esses dados foram analisados por estatística descritiva via programa Microsoft Excel (https://products.office.com/).

A abordagem qualitativa foi operacionalizada por meio de pesquisa de campo. Os cenários de estudo delimitados foram as regiões de saúde eleitas como objeto de investigação da pesquisa Política, Planejamento e Gestão das Regiões e Redes de Atenção à Saúde no Brasil, coordenada pela Faculdade de Medicina, Universidade de São Paulo, cujo objetivo é avaliar os processos de organização, coordenação e gestão envolvidos na conformação de regiões e redes de atenção à saúde As regiões foram as seguintes: Norte-Barretos (São Paulo), Rede Interestadual de Atenção à Saúde do Vale Médio São Francisco (Petrolina, Pernambuco e Juazeiro, Bahia - PEBA), entorno de Manaus e Alto Rio Negro (Amazonas), Baixada Cuiabana (Mato Grosso) e Região 10 - Capital e Vale do Gravataí (Rio Grande do Sul). Além dessas, foram investigadas três Regiões de Saúde do Estado do Rio de Janeiro: Norte Fluminense, Metropolitana I e Metropolitana II.

O universo da pesquisa correspondeu ao número total de cursos de graduação em Enfermagem, Farmácia, Fisioterapia, Medicina, Nutrição, Odontologia e Psicologia existentes e em atividade em 2013 nas regiões de saúde selecionadas, perfazendo 384 cursos 7.

Para determinação da amostra, de caráter intencional, foram considerados os gestores de ensino desses cursos (coordenador, vice-coordenador, diretor, chefe de departamento ou representante designado) disponíveis para participarem de uma entrevista in loco no período de coleta de dados determinado.

Considerando o tempo previsto de cinco dias de coleta de dados em cada uma das regiões, foram realizados convites prévios por telefone e/ou correio eletrônico, tendo-se a preocupação de abranger o maior número possível de cursos. Foram realizados 124 convites, dos quais $68(54,8 \%)$ foram aceitos mediante disponibilidade no período previsto para a coleta de dados. Dessa forma, foram realizadas entrevistas com 68 gestores de ensino de diferentes cursos de graduação em saúde, constituindo, portanto, a amostra final de cursos. A distribuição dos cursos por regiões de saúde consta na Tabela 1.

Os dados foram coletados por entrevistas in loco, a partir de um questionário semiestruturado, elaborado com base nas Diretrizes Curriculares Nacionais dos cursos de graduação em saúde, com 54 questões relativas ao perfil do entrevistado, caracterização da instituição de ensino e curso, estrutura curricular e perfil do egresso. Dadas a magnitude da pesquisa e a alta densidade dos resultados, ressalta-se que, neste artigo, serão apresentados apenas aqueles alinhados aos aspectos da regionalização, obtidos a partir de oito questões, referentes às estratégias adotadas (de inclusão e retenção de alunos e de abordagem regional do currículo) pelos cursos para o fortalecimento da região onde estão localizados e as mudanças verificadas no entorno em função das instituições.

\section{Tabela 1}

Número de cursos de graduação em saúde participantes da pesquisa, por regiões. Brasil, 2015 e 2016.

\begin{tabular}{lc}
\hline Regiões de Saúde & Número de cursos \\
\hline Norte-Barretos (São Paulo) & 5 \\
PEBA & 6 \\
Baixada Cuiabana (Mato Grosso) & 13 \\
Entorno de Manaus e Alto Rio Negro (Amazonas) & 9 \\
Região 10 - Capital e Vale do Gravataí (Rio Grande do Sul) & 5 \\
Norte Fluminense (Rio de Janeiro) & 14 \\
Metropolitana I (Rio de Janeiro) & 4 \\
Metropolitana II (Rio de Janeiro) & 68 \\
Total & 5
\end{tabular}

PEBA: Rede Interestadual de Atenção à Saúde do Vale Médio São Francisco (Petrolina, Pernambuco e Juazeiro, Bahia). Fonte: dados da pesquisa. 
As entrevistas foram gravadas, transcritas e submetidas à análise de conteúdo proposta por Bardin ${ }^{8}$. Essa técnica pressupõe três fases: (1) pré-análise, etapa na qual os depoimentos já transcritos foram organizados e submetidos à leitura flutuante; (2) exploração do material, em que foram destacados os recortes de ordem semântica do corpus de análise; (3) inferência e interpretação do material analisado.

Em atendimento às normas previstas na Resolução no 466/2012, do Conselho Nacional de Saúde, o projeto deste estudo foi submetido e aprovado pelo Comitê de Ética em Pesquisa do Instituto de Medicina Social da Universidade do Estado do Rio de Janeiro, sob parecer no 1.248.858.

\section{Resultados}

\section{Distribuição territorial da oferta de formação em saúde}

Em 2014, 1.107 instituições de Ensino Superior ofertavam 4.285 cursos de saúde. Desses, 72,1\% (n = 3.170) eram privados, e 2.225 (46,1\%) se localizavam na Região Sudeste do país.

Quanto às vagas, no ano 2000, foram ofertadas 164.103, passando a $884.568 \mathrm{em} \mathrm{2014,} \mathrm{o} \mathrm{que} \mathrm{cor-}$ responde a um aumento de 439\%. Nesse ano de 2014, São Paulo foi o estado que mais ofertou vagas em saúde ( $\mathrm{n}=51.692 ; 31,5 \%$ ), seguido pelo Rio de Janeiro ( $\mathrm{n}=19.364 ; 11,8 \%$ ) e por Minas Gerais ( $\mathrm{n}=14.277 ; 8,7 \%)$, isto é, três estados da Região Sudeste ofertaram mais da metade de todas as vagas do país. A Região Norte, por outro lado, foi a que ofertou menor número de cursos e vagas de graduação em saúde: $322(6,7 \%)$ e 43.380 (5,1\%), nessa ordem.

Em 2014, foram formados 152.333 profissionais de saúde, dos quais pouco mais de 72 mil (47,5\%) concluíram o curso em instituições localizadas no Sudeste. Apesar da concentração da oferta nessa região (Figura 1), no período 2000-2014, foi a Região Norte que apresentou as maiores taxas de incremento para cursos $(419,4 \%)$ e egressos $(557,1 \%)$, e a Região Nordeste, para vagas $(1.064,6 \%)$.

Figura 1

Distribuição de cursos, vagas e egressos de graduação em saúde, por grandes regiões. Brasil, 2000 e 2014.

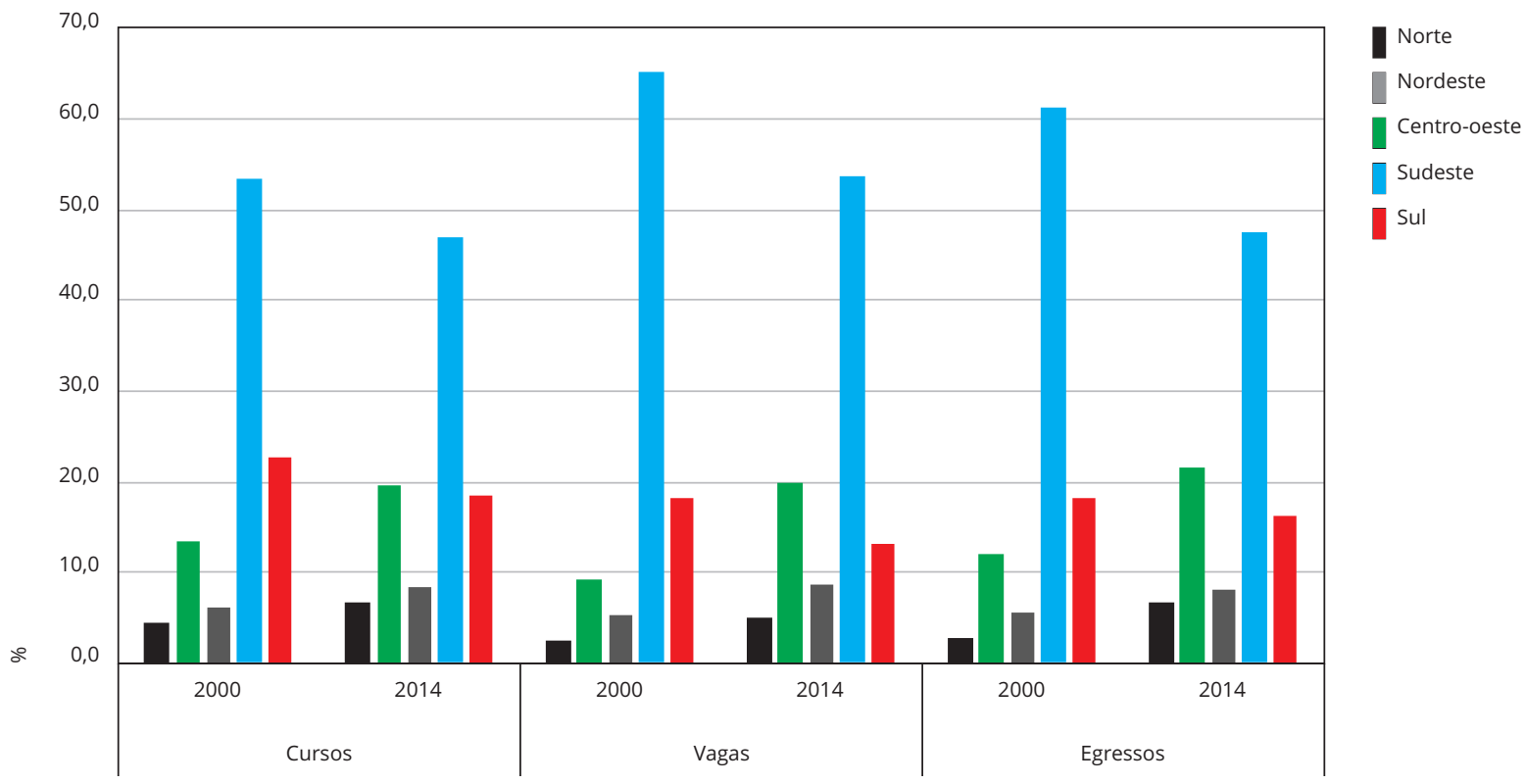

Fonte: Instituto Nacional de Estudos e Pesquisas Educacionais Anísio Teixeira (INEP) 7. 
Ao se verificarem os dados da Medicina e da Enfermagem desagregados por regiões de saúde, a diminuição da desigualdade na distribuição regional da oferta de formação em saúde se mostra mais evidente. No ano 2000, do total de 436 regiões de saúde, 62 ofertavam cursos de Medicina e 92 de Enfermagem, passando para 137 e 296, respectivamente, em 2014 (Tabela 2).

Embora a oferta desses cursos se dê essencialmente nas regiões metropolitanas de saúde com mais de 500 mil habitantes, a exemplo das Regiões de Saúde Metropolitana I (Rio de Janeiro) e São Paulo, as quais foram responsáveis pela maior oferta de cursos, vagas e egressos de Medicina e Enfermagem em 2014, o cálculo do incremento no período 2000-2014 apontou maiores taxas para as regiões de saúde com até 500 mil habitantes. Foi verificado aumento de 304,8\% de oferta de cursos de Medicina e de mais de 500\% de cursos de Enfermagem nas regiões com até 500 mil habitantes, ao passo que nas regiões com mais de 500 mil habitantes essas taxas foram inferiores a 250\% (Tabela 2).

Por meio da Tabela 3, é possível observar que, no ano 2000, em duas Regiões de Saúde que são cenários deste estudo, não havia cursos de Medicina. Da mesma forma, em três regiões, não havia cursos de Enfermagem. Em 2014, esses cursos foram ofertados em todas as regiões, representando, no período, um aumento de 52,9\% de cursos de Medicina e de 236,4\% de Enfermagem.

\section{Estímulo ao ingresso de alunos da região}

Entre os cursos investigados in loco, ações afirmativas têm promovido maior ingresso de alunos residentes na região onde se localiza a instituição de Ensino Superior. Entre os 30 cursos privados investigados, 21 aderem a programas de financiamento do Governo Federal ou têm uma política própria de auxílio estudantil. Entre os públicos, um federal e um estadual citaram cotas específicas para candidatos que residem em zonas próximas aos campi.

"Se nasce no Amazonas tem direito a um tipo de cota, se for do interior é outro tipo de cota; se for de fora do estado as chances são menores (...). A ideia é suprir o interior com profissionais, pois era muito comum o aluno de fora vir aqui se formar e depois voltar para a sua cidade" (Entorno de Manaus).

As modalidades de ingresso foram citadas pelos respondentes de todas as regiões como fator contributivo à entrada de alunos da região. O Exame Nacional do Ensino Médio (ENEM) foi relacionado como estratégia fundamental para a democratização do Ensino Superior, contribuindo para o maior acesso à graduação por alunos de baixo poder aquisitivo e residentes nos municípios do interior adjacentes ao local onde se encontram as instituições de Ensino Superior.

"Após a política de cotas atrelada ao ENEM, aumentou o número de alunos que têm por origem o município ou municípios vizinhos" (Metropolitana I/Rio de Janeiro).

\section{Tabela 2}

Oferta de cursos de graduação em Medicina e Enfermagem por grupamento de regiões de saúde, segundo o tamanho da população. Brasil, 2000 e 2014

\begin{tabular}{|c|c|c|c|c|c|c|}
\hline \multirow{2}{*}{$\begin{array}{l}\text { Classes de tamanho da população das Regiões de Saúde } \\
\text { (habitantes) }\end{array}$} & \multicolumn{3}{|c|}{ Medicina } & \multicolumn{3}{|c|}{ Enfermagem } \\
\hline & 2000 & 2014 & Incremento (\%) & 2000 & 2014 & Incremento (\%) \\
\hline Até 20.000 & 0 & 0 & - & 0 & 0 & - \\
\hline $20.001-50.000$ & 0 & 0 & - & 0 & 1 & - \\
\hline $50.001-100.000$ & 0 & 0 & - & 0 & 8 & - \\
\hline $100.001-500.000$ & 21 & 85 & 304,8 & 53 & 338 & 537,7 \\
\hline Acima de 500.000 & 78 & 160 & 105,1 & 131 & 503 & 284,0 \\
\hline n total de Regiões de Saúde & 62 & 137 & 121,0 & 92 & 296 & 221,7 \\
\hline n total de cursos ofertados & 99 & 245 & 147,5 & 184 & 850 & 362,0 \\
\hline
\end{tabular}

Fonte: Instituto Nacional de Estudos e Pesquisas Educacionais Anísio Teixeira (INEP) 7 . 


\section{Tabela 3}

Regiões de Saúde segundo número de municípios, população, proporção de médicos e enfermeiros por mil habitantes e número de cursos de Enfermagem e Medicina. Brasil, 2000, 2014 e 2015.

\begin{tabular}{|c|c|c|c|c|c|c|c|c|}
\hline \multirow[t]{2}{*}{ Regiões de Saúde } & \multirow[t]{2}{*}{$\begin{array}{l}\text { Número de } \\
\text { municípios }\end{array}$} & \multirow[t]{2}{*}{$\begin{array}{l}\text { População } \\
\text { em } 2014\end{array}$} & \multirow{2}{*}{$\begin{array}{c}\text { Médicos/mil } \\
\text { habitantes } \\
\text { em } 2015\end{array}$} & \multirow{2}{*}{$\begin{array}{c}\text { Enfermeiros/ } \\
\text { mil } \\
\text { habitantes } \\
\text { em } 2015\end{array}$} & \multicolumn{2}{|c|}{$\begin{array}{l}\text { Cursos de } \\
\text { Medicina }\end{array}$} & \multicolumn{2}{|c|}{$\begin{array}{c}\text { Cursos de } \\
\text { Enfermagem }\end{array}$} \\
\hline & & & & & 2000 & 2014 & 2000 & 2014 \\
\hline Entorno de Manaus (Amazonas) & 12 & 2.380 .116 & 1,36 & 0,87 & 1 & 3 & 2 & 9 \\
\hline PEBA & 17 & 1.013 .021 & 2,33 & 1,67 & 0 & 1 & 0 & 2 \\
\hline Metropolitana I (Rio de Janeiro) & 12 & 10.082.079 & 2,58 & 1,45 & 9 & 10 & 13 & 34 \\
\hline Metropolitana II (Rio de Janeiro) & 7 & 2.008 .412 & 2,34 & 1,00 & 1 & 1 & 3 & 5 \\
\hline Norte (Rio de Janeiro) & 8 & 882.578 & 2,31 & 1,26 & 1 & 2 & 0 & 7 \\
\hline Norte-Barretos (São Paulo) & 10 & 283.993 & 2,79 & 1,92 & 0 & 2 & 0 & 1 \\
\hline Região 10 - Capital e Vale do & 6 & 2.332 .573 & 3,64 & 1,64 & 3 & 4 & 2 & 10 \\
\hline \multicolumn{9}{|l|}{ Gravataí (Rio Grande do Sul) } \\
\hline Baixada Cuiabana (Mato Grosso) & 11 & 950.003 & 2,09 & 1,22 & 2 & 3 & 2 & 6 \\
\hline Conjunto das regiões & 83 & 19.932.775 & 2,49 & 1,36 & 17 & 26 & 22 & 74 \\
\hline
\end{tabular}

PEBA: Rede Interestadual de Atenção à Saúde do Vale Médio São Francisco (Petrolina, Pernambuco e Juazeiro, Bahia).

Fontes: Instituto Brasileiro de Geografia e Estatística (IBGE; http://www.ibge.gov.br), Instituto Nacional de Estudos e Pesquisas Educacionais Anísio

Teixeira (INEP) 7 .

\section{Estratégias curriculares com foco regional}

Verifica-se também que, além da ampliação da oferta de formação em saúde e de mais oportunidades de acesso na perspectiva da regionalização, o desenvolvimento da região é assumido como bandeira de algumas instituições, conforme depoimento de gestores da Norte Fluminense, PEBA, Norte-Barretos, Baixada Cuiabana e Entorno de Manaus. Para isso, algumas estratégias curriculares vêm sendo adotadas, como o fortalecimento de pesquisas, de atividades com a comunidade e de conteúdos curriculares que tenham por foco os problemas e as características da região.

"Criamos uma clínica de psicologia para ampliarmos o atendimento de saúde mental da população, em especial dos trabalhadores transitórios e que trabalham nas plataformas petroliferas da região que têm alto indice de problemas psiquiátricos, pela distância das famílias, pelo trabalho confinado" (Norte Fluminense/ Rio de Janeiro).

A maior parte dos respondentes afirmou, no entanto, que o esforço das instituições em dar foco às necessidades locais esbarra em inúmeras dificuldades, entre elas as de caráter logístico para acesso a áreas geograficamente menos acessíveis, como comunidades ribeirinhas e indígenas (Entorno de Manaus e Baixada Cuiabana), e para acesso a áreas socialmente mais vulneráveis, como as comunidades dominadas por facções criminosas, que põem em risco a segurança dos alunos e docentes (Metropolitanas I e II/Rio de Janeiro).

Outro ponto abordado por todos os gestores de ensino entrevistados se refere à ausência, à insuficiência e/ou às deficiências estruturais dos aparelhos de saúde utilizados como campos de estágio, o que dificulta a vivência dos alunos no SUS e o desenvolvimento de competências profissionais alinhadas à realidade locorregional.

"Eu acho que o nosso maior problema é o acesso ao SUS, especialmente por causa da infraestrutura das unidades básicas, que são muito pequenas e precárias" (Norte-Barretos/São Paulo).

Nessa mesma linha, a pouca valorização das atividades de extensão distancia a universidade da comunidade e, por consequência, enfraquece essa relação, que tem potencial para transformar a realidade, como mencionado por entrevistado da Região Metropolitana II. 


\section{Fixação profissional na região}

Em se tratando da fixação profissional, o investimento direto das instituições de ensino das regiões de saúde Entorno de Manaus, Norte Fluminense, Baixada Cuiabana, Norte-Barretos e PEBA se reduz a ofertar cursos de pós-graduação, no entendimento de que este é o fator mais importante para a retenção de profissionais de saúde, em especial de médicos. Os entrevistados reconhecem, no entanto, que a infraestrutura local e as perspectivas salariais contribuem para a decisão dos egressos de ficar ou de migrar para outros locais.

Sobre o assunto, com exceção dos gestores de ensino vinculados a cursos da região Norte Fluminense, todos os demais informam que uma parcela considerável dos egressos tem permanecido na região após a formatura. Deve-se destacar, contudo, que a maioria desses egressos tem origem na própria região. O contrário ocorre nos casos em que os alunos são de fora, que tendem a voltar para seus locais de origem ou se deslocam para municípios de maior porte ou próximo a eles, de modo que a fixação profissional não se constitui grande preocupação para as instituições de ensino das Regiões Metropolitanas.

"A maioria quer ficar onde tem mais recurso. E é na capital. Mas eles têm ficado em outros municípios da Região Metropolitana” (Região 10/Rio Grande do Sul).

\section{A universidade como propulsora do desenvolvimento regional e das regiões de saúde}

Para os respondentes do Entorno de Manaus, Baixada Cuiabana e PEBA, a presença da universidade tem desencadeado inúmeros processos de mudança estrutural que têm impulsionado o desenvolvimento da região.

"Na época da criação da universidade não se tinham apartamentos suficientes aqui, o setor imobiliário, então, cresceu com a chegada de alunos e de professores, que vieram todos de fora. (...) Também mudou o conceito de fazer assistência na região: com o academicismo dos professores, a assistência passou a se basear em evidências e não mais em achismos e intuição" (PEBA).

"Se ela [universidade] não existisse aqui, nós não teríamos como mostrar ao mundo a nossa biodiversidade, o nosso conhecimento, a magnitude da Amazônia. A ciência que é desenvolvida aqui é impar. (...) temos pesquisadores de renome" (Entorno de Manaus).

"Ela atua em todas as áreas, formando profissionais, (...) ajudando os municípios a planejar o saneamento (...). Ela mudou o estado para ele ser hoje um campeão na agricultura de soja, de algodão, de gado. No campo da pesquisa, vem desenvolvendo seleção genética animal, produzindo sementes resistentes (...). Ela mudou a formação das pessoas (...), ela garante a possibilidade de pensarmos e repensarmos a situação regional. (...) Muitas pessoas vieram de fora do estado para cursar essa universidade e se fixaram; estão trabalhando e se reproduzindo" (Baixada Cuiabana/Mato Grosso).

Com relação ao impacto das instituições de ensino superior no desenvolvimento das redes de atenção à saúde, e propriamente das regiões de saúde, evidenciou-se que os cenários metropolitanos estudados aqui (Metropolitanas I e II e Região 10) apresentam dificuldades de estruturação de uma rede regional. Para os respondentes, isso ocorre principalmente devido à concentração de unidades de saúde de maior complexidade nesses locais e que operam de modo desarticulado das unidades básicas. Essas, por sua vez, são insuficientes para ordenar as demandas.

"A cobertura da atenção básica ainda está muito aquém do que se precisa. Então, ela não está cumprindo o seu papel de ordenador do SUS. Aqui, o maior aporte é de hospitais gerais e especializados que pouco se comunicam com a saúde da família" (Metropolitana II/Rio de Janeiro).

Na Região de Saúde Norte-Barretos, os cursos da área da saúde foram criados para acolher as demandas do Hospital do Câncer de Barretos (atualmente Hospital de Amor), cuja expansão desencadeou o processo de desenvolvimento econômico local, mas pouco contribuiu para o desenvolvimento da rede de saúde, segundo os depoentes. Há um investimento importante em pesquisa clínica e formação de recursos humanos em saúde, com apoio de financiamento público e privado, mas que se centra na atenção especializada. Para os gestores de ensino, os problemas básicos de saúde locais não estão incluídos na agenda de governo. Há um sentimento de que a Fundação Pio XII, mantenedora do hospital, sobrecarrega o SUS de Barretos e, com isso, restringe a oferta de serviços de saúde primários para o cidadão local. Também foi referida dificuldade de contratualização de convênio entre o SUS 
e as instituições de ensino que fornecem atendimento de saúde gratuito à população, caso de uma clínica de odontologia de curso privado cujos custos são repassados aos estudantes.

"A clínica faz atendimento gratuito à população e, por isso, o curso tem grande importância regional. Mas o atendimento está prejudicado, pois não temos convênio com o SUS, embora tenhamos feito inúmeras tentativas de conversa com a rede municipal" (Norte-Barretos/São Paulo).

O destaque é PEBA, em que a universidade, nesse caso, a federal, provocou e tem provocado maiores impactos na estruturação de uma rede de saúde, conforme pontuado pelos entrevistados. Nessa região, com a implantação da universidade e a oferta de cursos da área da saúde, acompanhada pelo desenvolvimento econômico da região, equipamentos foram requeridos. Nesse movimento, a Prefeitura de Petrolina inaugurou um hospital municipal que logo passou a ser administrado pela esfera federal, tornando-se hospital universitário ligado à universidade federal e campo de estágio das instituições de ensico superior localizadas no entorno. É, hoje, a unidade de referência para os 53 municípios de PEBA. Mais recentemente, uma policlínica ligada à universidade federal foi inaugurada. A região passa a contar, então, com equipamentos de saúde, programas de residência e pós-graduação, especialistas de diversas áreas, além de projetos de pesquisa e extensão voltados ao conhecimento, manutenção e preservação ambiental.

Outro ponto a destacar em PEBA é a criação de novos campi em municípios da região, em especial o de Paulo Afonso, que implantou o curso de Medicina no contexto do PMM, o que pode contribuir para o fortalecimento da rede de atenção regional.

"A universidade meio que organiza a rede, que é bem robusta. Envolve dois estados, 53 municípios, 2 milhões e 100 mil habitantes... (...) essa rede é muito útil para a região. (...) Temos o hospital universitário, os consultórios itinerantes de oftalmologia e odontologia que vão nos bairros, nas escolas. Temos a policlínica, residência (...). E, em Paulo Afonso, estamos tentando incorporar o hospital de lá para ser universitário. Ele é um campus muito estratégico porque alcança parte da população que está longe daqui da sede” (PEBA).

\section{Discussão}

Os dados apresentados sobre a distribuição da oferta de formação em saúde apontam crescimento da disponibilidade de cursos, vagas e egressos, mas esse crescimento ainda se mantém concentrado nos locais mais desenvolvidos. Não obstante, percebe-se maior difusão dessa oferta entre regiões, regiões de saúde e municípios menos favorecidos em termos socioeconômicos, logísticos e estruturais. Essa maior distribuição veio acompanhada justamente de maior investimento econômico e social nesses locais, pelas esferas públicas e privadas, o que desencadeou, por conseguinte, aumento do produto interno bruto e da renda familiar, além de geração de empregos 9,10 .

Políticas específicas de interiorização do ensino superior podem ter contribuído para a mudança no panorama de distribuição da oferta de formação em saúde. Nessa perspectiva, pode-se citar o Programa de Apoio a Planos de Reestruturação e Expansão das Universidades Federais (Reuni), que elevou o número de municípios atendidos por universidades federais em 138\%, no período 2003 2010. Como incentivo à integração e ao desenvolvimento regional, novas instituições federais foram criadas em locais estratégicos, como a Universidade Federal da Fronteira Sul (que integra os estados fronteiriços da região Sul do Brasil), a Universidade Federal do Oeste do Pará (que integra a Região Amazônica) e a Universidade Federal do Sul da Bahia 11.

A ampliação da oferta, no entanto, não garante necessariamente maior democratização do acesso ao ensino e, por isso, ações afirmativas também se destacam por agenciar não apenas oportunidades mais equânimes de entrada à universidade, mas também a permanência de estudantes em situações de vulnerabilidade social. Exemplos são o Programa Nacional de Assistência Estudantil (Pnaes) 12, o Programa Universidade para Todos (Prouni) 13 e o Programa de Financiamento Estudantil (Fies) 14.

Neste estudo, constatou-se que as instituições investigadas adotam estratégias semelhantes, com vistas a promover o ingresso de estudantes residentes no entorno, o que inclui programas de financiamento, cotas específicas e modalidades de ingresso diferenciadas, entre elas o ENEM.

Alguns fatores podem justificar a influência do ENEM nessa dinâmica. Entre esses fatores, está o aumento do número de municípios que sediam a realização das provas, já que, com o sistema de vestibular, as universidades aplicam as provas nas cidades onde se localizam os campi e em outros poucos 
municípios do próprio estado. Outros fatores associados são o sistema de cotas e os programas de financiamento estudantil vinculados ao ENEM 15.

Ainda nessa linha, os respondentes das regiões Norte e Nordeste informaram que as instituições têm empreendido esforços no desenvolvimento de programas de pós-graduação como recurso para a fixação profissional na região. Reconhecem, entretanto, que a pós-graduação por si só é insuficiente para reter os egressos e sugerem outros fatores relacionados, como a infraestrutura local, as condições de vida e de trabalho e também as perspectivas de emprego e salário, tal como evidenciado por estudos sobre o tema 16,17 .

Com base nas análises, é importante pontuar que, embora se verifique a permanência de egressos no entorno das universidades, os que se formam em regiões metropolitanas ou muito próximas a elas tendem a se fixar ou a buscar fixação nas capitais ou em municípios de maior desenvolvimento econômico, o que ratifica que a maior dificuldade não é a de reter o profissional no estado ou na região de saúde, mas no interior e em áreas mais remotas 18,19,20,21.

Esse contexto demonstra a relevância da presença de cursos de maneira menos concentrada nas grandes capitais e regiões, não apenas porque significaria promover a retenção profissional no interior, mas sobretudo porque a presença de aparelhos formadores, em especial de universidades de maior porte, como as federais, tende a agenciar o desenvolvimento da região 22 . Cria-se, nesse entendimento, um processo de retroalimentação, em que as instituições formadoras atuam como mobilizadoras e, ao mesmo tempo, favorecidas do desenvolvimento regional.

O desenvolvimento regional pode ser compreendido como um conjunto de ações que busca combinar e utilizar eficientemente as potencialidades territoriais a políticas setoriais e regionais, para fornecer a reestruturação produtiva e a mudança estrutural da economia local e, por consequência, estabelecer e disponibilizar bens e serviços básicos em condições de acesso para toda a população local. Implica, então, a oferta de infraestrutura para o funcionamento do sistema produtivo, como transportes, escolas e unidades de saúde; a oferta de condições imateriais, como os conhecimentos práticos em diversas áreas; a capacidade de organização regional; e a posse e o uso de instrumentos financeiros e de utilização de recursos naturais existentes 23.

Nessa concepção, acredita-se que a universidade é um importante fator propulsor do desenvolvimento regional, por fornecer sua expertise, fortalecer a governança local, e por aproximar-se da sociedade civil, dando-lhe condições de contribuir com ações desenvolvimentistas 24 , como pontuado pelos respondentes deste estudo. Segundo eles, iniciativas curriculares têm sido desenvolvidas pelas instituições de ensino na perspectiva de potencializar o desenvolvimento regional e de preparar os futuros egressos para atuarem conforme a realidade sociossanitária local. Por outra via, dificuldades logísticas e de aparelhamento da rede de saúde constituem-se desafios para a qualificação do ensino consoante as demandas regionais.

O processo de regionalização e interiorização do ensino superior pressupõe a multiplicação de infraestrutura, recursos humanos e instituições de apoio à formação em locais desprovidos ou com baixa oferta desses atributos 25 . Nesse movimento, equipa-se o "entorno universitário", que acaba por se constituir local atrativo para o estabelecimento da população. Mais ainda, a universidade avaliza a participação da região no uso dos recursos nacionais, contribuindo para o fortalecimento da capacidade de organização social da região. O desenvolvimento de pesquisas, por exemplo, contribui significativamente para o surgimento de indústrias, novos produtos e tecnologias, com efeitos na economia local 18, conforme pontuado por parte dos gestores de ensino.

Essas contribuições vão ao encontro do que apregoa a Resolução no 569/2017, a qual ratifica a responsabilidade social das instituições de Ensino Superior com o seu entorno e o compromisso com a promoção do desenvolvimento de redes assistenciais e com a formação de recursos humanos em saúde que possibilitem a transformação da realidade em que estão inseridas 26.

Especificamente sobre o desenvolvimento das redes e Regiões de Saúde, verificou-se que as instituições de ensino das regiões metropolitanas mostram-se menos potentes. Isso se deve, sobretudo, à concentração da capacidade instalada nas capitais dos estados de que fazem parte e ao histórico de centralização de poder decisório pela esfera estadual, cujo desenvolvimento econômico ocasionou condições institucionais assimétricas para a descentralização entre os municípios 27. Outros fatores associados são a baixa cobertura da atenção primária e a sua desvinculação da política regional, em contraste com o elevado número de equipamentos terciários, bem como a presença de inúmeras insti- 
tuições que operam no sistema de saúde sob a forma de contratos e convênios, a exemplo das empresas que intermedeiam a contratação de recursos humanos em saúde para os serviços de saúde 28 .

De maneira contrária, as instituições de ensino de PEBA, em especial a federal, têm sido fundamentais para a estruturação e qualificação da rede de saúde. Cabe destacar que os desdobramentos em função dessa universidade federal têm completa coerência com a proposta de sua criação, segundo a qual os principais desafios da instituição seriam o de oferecer um ensino de qualidade para formar profissionais competentes e gerar tecnologias adequadas ao contexto, tendo por missão o desenvolvimento socioeconômico da região ${ }^{29}$. Nessa lógica, a universidade federal tem se expandido para o interior da região a partir da abertura de novos cursos, a exemplo do curso de Medicina criado no bojo do PMM.

A proposta desse programa vem a reboque de uma discussão histórica, iniciada sobretudo nos anos 2000, quando se constatou um desequilíbrio numérico e distributivo de recursos humanos em saúde no mercado de trabalho ${ }^{9}$. Esse contexto implicou a necessidade de se adotarem medidas governamentais de fomento à abertura de cursos de saúde em áreas desprovidas de assistência, como o caso do PMM, que também envolve políticas de atração profissional, de expansão de vagas de residência em áreas prioritárias e estruturação da rede assistencial. Na perspectiva de impulsionar a reorientação da formação médica, novas Diretrizes Curriculares Nacionais foram instituídas, as quais enfatizam a inserção locorregional e a integração dos cursos com a rede de serviços de saúde 30,31.

A expectativa é que o PMM supere os obstáculos evidenciados em políticas adotadas anteriormente e provoque a revisão das Diretrizes Curriculares Nacionais dos demais cursos da saúde, na perspectiva de reafirmar a necessidade de readequação de perfil profissional às necessidades de saúde da população e de garantir uma formação comprometida com a superação das iniquidades que causam o adoecimento das coletividades regionais 26 .

Pelo exposto, apreende-se que, em todas as regiões de saúde analisadas, há instituições de ensino que adotam estratégias de estímulo ao ingresso de alunos do entorno. As voltadas para a fixação profissional, por sua vez, foram mencionadas apenas pelos representantes de cursos da Norte Fluminense, PEBA, Norte-Barretos, Baixada Cuiabana e Entorno de Manaus. Também foi nessas regiões que se identificaram iniciativas curriculares com foco regional, mas as dificuldades para operacionalizá-las foram apontadas por todos os 68 respondentes.

O desenvolvimento regional em função da presença de instituições de ensino foi descrito pelos depoentes do Entorno de Manaus, Baixada Cuiabana e PEBA. A universidade federal dessa última região demonstra, ainda, ser importante fator de ordenamento da rede de saúde regional.

Por seu caráter exploratório, este estudo abre possibilidades para o desenvolvimento de outras pesquisas que tentem superar as limitações deste estudo, por ocasião do desenho metodológico adotado. Uma delas diz respeito ao foco nas opiniões de apenas um ator imbricado no processo de formação de recursos humanos em saúde que, como porta-voz de sua instituição de ensino, pode ter se debruçado mais sobre os pontos positivos dela. Nesse sentido, esta pesquisa é insuficiente para a apreensão da complexidade que envolve o papel das instituições de ensino no processo de regionalização. A ausência de pesquisas documentadas sobre o objeto em análise pode ser também reconhecida como um limitante.

\section{Conclusões}

É fato que não se pode contextualizar o processo de regionalização sem a análise aprofundada das diversas dimensões que a afetam, como a governança, a política, os processos organizativos de oferta e de fluxos de demanda da saúde, a incorporação tecnológica, a gestão clínica e os recursos humanos. Assim, este artigo não pretendeu condicionar o desenvolvimento das regiões de saúde à presença de instituições de ensino com oferta de formação de profissionais de saúde, mas delinear a sua importância para esse processo.

Verificou-se que, apesar da concentração na região Sudeste, houve um aporte distributivo de instituições formadoras e vagas de cursos de graduação em saúde para regiões do Norte e Nordeste, a exemplo do Entorno de Manaus e PEBA, que obedecem às características regionais. Verificou-se também que as políticas de acesso ao Ensino Superior, de ingresso e de manutenção de estudantes da 
região, bem como as estratégias de fixação cunhadas pelas instituições de Ensino Superior têm grande relevância para o fortalecimento das Regiões de Saúde.

Os resultados apresentados destacam a importância das instituições de ensino no fortalecimento da regionalização, no aporte ao desenvolvimento tecnológico e na expansão do mercado de trabalho, modificando o complexo produtivo local, especialmente as regiões anteriormente desprovidas desses equipamentos. Por outra via, apontam para a necessidade de acompanhamento desse panorama, com o intuito de verificar a permanência e a sustentabilidade dessas instituições e de seu papel contributivo para o desenvolvimento regional e, sobretudo, para a diminuição das desigualdades de oferta de profissionais e de serviços de saúde.

\section{Colaboradores}

C. R. Pierantoni participou da concepção e projeto, interpretação dos dados, revisão crítica relevante do conteúdo intelectual, aprovação final da versão a ser publicada, é responsável por todos os aspectos do trabalho na garantia da exatidão e integridade de qualquer parte da obra. C. Magnago, S. P. Vieira e M. S. Ney contribuíram na concepção e projeto, análise e interpretação dos dados, redação do artigo, aprovação final da versão a ser publicada e são responsáveis por todos os aspectos do trabalho na garantia da exatidão e integridade de qualquer parte da obra. R. G. Miranda e S. N. Girardi colaboraram na concepção e projeto, análise e interpretação dos dados, aprovação final da versão a ser publicada e são responsáveis por todos os aspectos do trabalho na garantia da exatidão e integridade de qualquer parte da obra.

\section{Informações adicionais}

ORCID: Celia Regina Pierantoni (0000-0001-74816350); Carinne Magnago (0000-0001-8799-3225); Swheelen de Paula Vieira (0000-0002-0135-1860); Márcia Silveira Ney (0000-0002-3262-565X); Rômulo Gonçalves Miranda (0000-0001-80823768); Sabado Nicolau Girardi (0000-0003-08170533).

\section{Agradecimentos}

Agradecimentos à equipe da Pesquisa Política, Planejamento e Gestão das Regiões e Redes de Atenção à Saúde no Brasil (Região e Redes), especialmente à Profa Dra. Ana Luiza d'Ávila Viana, e à equipe da Estação de Pesquisa de Sinais de Mercado, Núcleo de Estudos em Saúde Coletiva, Universidade Federal de Minas Gerais, parceiras no desenvolvimento da pesquisa da qual se originou este manuscrito. Resultados de pesquisa financiada por Carta Acordo com a Organização Pan-Americana da Saúde (OPAS).

\section{Referências}

1. Duarte CMR, Pedroso MM, Bellido JG, Moreira RS, Viacava F. Regionalização e desenvolvimento humano: uma proposta de tipologia de Regiões de Saúde no Brasil. Cad Saúde Pública 2015; 31:1163-74.

2. Mendes EV. As redes de atenção à saúde. Ciênc Saúde Colet 2010; 15:2297-305.

3. Viana ALD, Lima LD, organizadores. Regionalização e relações federativas na política de saúde no Brasil. Rio de Janeiro: Contracapa; 2011.

4. Magnago C, Pierantoni CR, Carvalho CL, Girardi SN. Nurse training in health in different regions in Brazil. Rev Bras Saúde Mater Infant 2017; 17 Suppl 1:S219-28.

5. Batista CB. Movimentos de reorientação da formação em saúde e as iniciativas ministeriais para as universidades. Barbaroi 2013; (38):97125.

6. Gadelha CAG, Costa LS. A saúde na política nacional de desenvolvimento: um novo olhar sobre os desafios da saúde. In: Fundação Oswaldo Cruz, organizador. A saúde no Brasil em 2030 - prospecção estratégica do sistema de saúde brasileiro: desenvolvimento, Estado e políticas de saúde. v. 1. Rio de Janeiro: Fundação Oswaldo Cruz/Instituto de Pesquisa Econômica Aplicada/Brasília: Secretaria de Assuntos Estratégicos da Presidência da República/Ministério da Saúde; 2013. pp. 103-32.

7. Instituto Nacional de Estudos e Pesquisas Educacionais Anísio Teixeira. Censo da educação superior 2013. Brasília: Instituto Nacional de Estudos e Pesquisas Educacionais Anísio Teixeira; 2015.

8. Bardin L. Análise de conteúdo. São Paulo: Edições 70; 2011.

9. Pierantoni R, Magnago C. Tendências das graduações em saúde no Brasil: análise da oferta no contexto do Mercosul. Divulg Saúde Debate 2017 ; $57: 30-43$. 
10. Albuquerque MV, Viana ALD, Lima LD, Ferreira MP, Fusaro E, Iozzi F. Regional health inequalities: changes observed in Brazil from 2000-2016. Ciênc Saúde Colet 2017; 22:1055-64.

11. Ministério da Educação. Análise sobre a expansão das universidades federais 2003 a 2012. Relatório da Comissão Constituída pela Portaria no 126/2012. Brasília: Ministério da Educação; 2012.

12. Brasil. Decreto no 7.234 , de 19 de julho de 2007. Dispõe sobre o Programa Nacional de Assistência Estudantil - PNAES. Diário Oficial da União 2010; 20 jul.

13. Brasil. Lei no 11.096, de 13 de janeiro de 2005. Institui o Programa Universidade para Todos - PROUNI, regula a atuação de entidades beneficentes de assistência social no ensino superior; altera a Lei no 10.891 , de 9 de julho de 2004, e dá outras providências. Diário Oficial da União 2005; 14 jan.

14. Brasil. Lei no 10.260 , de 12 de julho de 2001 . Dispõe sobre o Fundo de Financiamento ao estudante do Ensino Superior e dá outras providências. Diário Oficial da União 2001; 13 jul.

15. Mello Neto RD, Medeiros HAV, Paiva FS, Simões JL. O impacto do Enem nas políticas de democratização do acesso ao Ensino Superior Brasileiro. Comunicações 2014; 21:109-23.

16. Stralen ACSV, Massote AW, Carvalho CL, Girardi SN. Percepção de médicos sobre fatores de atração e fixação em áreas remotas e desassistidas: rotas da escassez. Physis (Rio J.) 2017; 27:147-72.

17. Ney MS, Rodrigues PHA. Fatores críticos para a fixação do médico na Estratégia Saúde da Família. Physis (Rio J.) 2012; 22:1293-311.

18. Goma FM, Tomblin Murphy G, MacKenzie A, Libetwa M, Nzala SH, Mbwili-Muleya C, et al. Evaluation of recruitment and retention strategies for health workers in rural Zambia. Hum Resour Health 2014; 12 Suppl 1:S1.

19. Lee DM, Nichols T. Physician recruitment and retention in rural and underserved areas. Int J Health Care Qual Assur 2014; 27:642-52.

20. Targa LV, Silva AL, Silva DHS, Barros EF, Schwalm FD, Savassi LCM, et al. Declaração de Belém: o recrutamento e a retenção de profissionais de saúde em áreas rurais e remotas. Rev Bras Med Fam Comunidade 2014; 9:64-9.
21. World Health Organization. Increasing access to health workers in remote and rural areas through improved retention: global policy recommendations. Geneva: World Health Organization; 2010.

22. Oliveira Jr. A. A universidade como polo de desenvolvimento local/regional. Caderno de Geografia 2014; 24:1-12.

23. Costa Silva J. Compêndio de economia regional. Paredes: Principia; 2009.

24. Lara JC, Carniello MF. Desenvolvimento regional e a expansão do ensino superior público: o caso da Universidade do Estado de Mato Grosso. Revista de Ensino Educação e Ciências Humanas 2018; 19:52-8.

25. Vargas HM. Interiorização da educação superior pública no Brasil: pontos de atenção, ajustes e autonomia universitária. Revista de Estudios Brasileños 2016; 3:156-69.

26. Conselho Nacional de Saúde. Resolução no 569, de 8 de dezembro de 2017. Diário Oficial da União 2018; 26 fev.

27. Albuquerque MV. O enfoque regional na política de saúde brasileira (2001-2011): diretrizes nacionais e o processo de regionalização nos estados brasileiros [Tese de Doutorado]. São Paulo: Universidade de São Paulo; 2013.

28. Viana ALD, Ferreira MP, Cutrim MAB, Fusaro ER, Souza MR, Mourão L, et al. The regionalization process in Brazil: influence on policy, structure and organization dimensions. Rev Bras Saúde Mater Infant 2017; 17 Suppl 1: S27-43.

29. Universidade Federal do Vale do São Francisco. Plano de Desenvolvimento Institucional PDI 2009-2014. Petrolina: Universidade Federal do Vale do São Francisco; 2009.

30. Brasil. Lei no 12.872 , de 22 de outubro de 2013. Institui o Programa Mais Médicos, altera as Leis no 8.745, de 9 de dezembro de 1993 , e no 6.932 , de 7 de julho de 1981, e dá outras providências. Diário Oficial da União 2013; 23 out.

31. Oliveira APC, Dal Poz MR, Craveiro I, Gabriel M, Dussault G. Factors that influence human resources for health policy formulation: a multiple case study in Brazil and Portugal. Cad Saúde Pública 2018; 34:e00220416. 


\section{Abstract}

This article aims to provide an overview of the territorial distribution of health training supply and to identify the strategies for strengthening regionalization adopted by teaching institutions that offer health courses and the changes in the regional surroundings as a function of these institutions. This is a descriptive-exploratory multiple-case study conducted at the national level in Brazil from December 2015 to September 2016. The study analyzed secondary data on health training supply and interviewed 68 administrators of undergraduate health courses, whose commentary was submitted to content analysis. There was an increase in health training institutions in smaller regions and municipalities (counties), although such institutions were still concentrated mainly in more socioeconomically developed regions. Policies have been implemented for access and enrollment in higher education from the perspective of promoting provision and professional retention in the region where the health course is located. The university's presence promotes regional development and has the potential to strengthen health regionalization.

Regional Health Planning; Health Manpower; Higher Education; Universities

\section{Resumen}

El objetivo de este artículo es describir el panorama de la distribución territorial, en cuanto a la oferta de formación en salud, e identificar estrategias para el fortalecimiento de la regionalización, adoptadas por las instituciones de enseñanza que ofrecen cursos de salud, además de analizar los cambios verificados en el entorno regional, en función de estas instituciones. Se trata de un estudio descriptivo-exploratorio, de casos múltiples, desarrollado en el ámbito nacional de Brasil durante el período de diciembre de 2015 a septiembre de 2016. Se analizaron datos secundarios de oferta de formación en salud, y se entrevistaron a 68 gestores de enseñanza de cursos de grado en salud, cuyas declaraciones fueron sometidas a análisis de contenido. Se percibe un aumento de equipamientos para la formación en salud en regiones $y$ municipios de menor porte, pese a la concentración en lugares con mayor desarrollo socioeconómico. Se están emprendiendo politicas de acceso e ingreso en la enseñanza superior de estudiantes, desde la perspectiva de promover la provisión y emplazamiento del profesional en el entorno donde se localizan los cursos de salud. Se constata que la presencia de la universidad promueve el desarrollo regional y tiene potencial para el fortalecimiento de la regionalización de la salud.

Regionalización; Recursos Humanos en Salud; Educación Superior; Universidades
Recebido em 05/Abr/2018

Versão final reapresentada em 06/Ago/2018

Aprovado em 17/Ago/2018 